\title{
COVID-19 y enfermedad renal crónica: ¿qué debemos saber sobre la relación ECA/ECA-2?
}

\section{COVID-19 and chronic kidney disease: what should we know about ACE/ACE-2 ratio?}

\author{
Cristhian Adolfo Vizcarra-Vizcarra'
}

\author{
Vizcarra-Vizcarra C. COVID-19 y enfermedad renal crónica: ¿qué \\ debemos saber sobre la relación ECA/ECA-2?. Rev Soc Peru \\ Med Interna. 2020;33(4): 155-160. \\ https://doi.org/10.36393/spmi.v33i4.563
}

\begin{abstract}
RESUMEN
La infección viral por el coronavirus SARS-CoV-2 produce una enfermedad denominada COVID-19, caracterizada por un síndrome respiratorio agudo grave que puede progresar a falla multiorgánica y muerte. Los pacientes que cursan con dicha entidad, comúnmente, presentan diversas comorbilidades, entre las que resalta la enfermedad renal crónica, la que podría estar relacionada a infección severa. La relación ECA/ECA2 elevada, juega un papel importante en la fisiopatología de la enfermedad renal crónica y en diferentes enfermedades cardiovasculares, predisponiendo a estos pacientes a desarrollar la infección con mal pronóstico. Hasta un $46 \%$ de los pacientes en hemodiálisis con COVID-I9 son asintomáticos, aunque algunos cursan con fiebre, tos y disnea, y es común observar síntomas gastrointestinales que deben ser tomados en cuenta al evaluar a este grupo de pacientes en las unidades de diálisis.
\end{abstract}

Palabras claves: COVID-19, ECA-2, enfermedad renal crónica, hemodiálisis.

\begin{abstract}
Viral infection with the SARS-CoV-2 coronavirus produces a disease called COVID-19, characterized by a severe acute respiratory syndrome that can progress to multiple organ failure and death. Patients who have this entity commonly present various comorbidities, among which chronic kidney disease stands out, which could be related to severe infection. High levels of ACE / ACE - 2 ratio plays an important role in the pathophysiology of chronic kidney disease and in different cardiovascular diseases, predisposing these patients to develop infection with a poor prognosis. Up to $46 \%$ of hemodialysis patients with COVID are asymptomatic, although some present with fever, cough, and dyspnea, and it is common to observe gastrointestinal symptoms that must be taken into account when evaluating this group of patients in dialysis units.
\end{abstract}

Keywords: COVID - 19; ACE - 2; Chronic kidney disease; Hemodialysis

Médico nefrólogo. Centro Nefrológico Arequipa, Perú.

\section{INTRODUCCIÓN}

La COVID-19, una nueva enfermedad generada por el coronavirus SARS-CoV-2, puede producir un síndrome respiratorio agudo grave (SARS) con sepsis, falla multiorgánica y muerte, y ha provocado una catástrofe mundial. Se inició en Wuhan, China, en diciembre del 2019, donde se reportaron casos de neumonía atípica que por su alta velocidad de propagación ha generado 21294 845 infectados y 761779 fallecidos a nivel mundial al 16 de agosto. ${ }^{1}$

El SARS-CoV-2 ingresa al organismo a través de las vías respiratorias mediante la unión de su proteína espiga o spike (S) al receptor de la enzima convertidora de angiotensina-2 (ECA-2), presente en las células pulmonares, del intestino, corazón, riñón y testículos. La ECA-2 participa en la conversión de angiotensina I a angiotensina 1-9 y de angiotensina II a angiotensina 1-7, con efecto antagónico y contrarregulador de la enzima convertidora de angiotensina, produciendo vasodilatación, antiproliferación celular y disminuyendo la fibrosis e hipertrofia ${ }^{(2)}$.

Los enfermos con COVID-19 presentan diferentes comorbilidades. Un estudio en Wuhan mostró que la 
hipertensión y la diabetes fueron las patologías más frecuentes $(15 \%$ y $7,4 \%$, respectivamente), mientras que otro estudio resaltó a la hipertensión arterial $(56,6 \%)$, la obesidad $(41,7 \%)$, la diabetes mellitus $(33,8 \%)$ y la enfermedad renal crónica terminal $(3,5 \%)$ como las enfermedades preexistentes más comunes en este grupo de pacientes. ${ }^{3,4}$

Se realizó una búsqueda en PubMed de los trabajos publicados considerando a los términos "COVID-19", "Coronavirus", "SARS-CoV-2" y "Diálisis", encontrando pocos estudios publicados y algunos reportes de casos, por lo que se realizó la presente revisión.

\section{COVID-19 Y ENFERMEDAD RENAL CRÓNICA}

Pocos estudios hablan sobre la interacción entre COVID-19 y la enfermedad renal crónica, enfocando esta, principalmente, como una comorbilidad hallada en los pacientes que desarrollan la infección, con frecuencias que oscilan entre el $0,7 \%$ y $38,0 \% .^{3-7}$ Sin embargo, un estudio encontró que la enfermedad renal se asocia a una tasa significativamente mayor de muerte hospitalaria en pacientes con COVID-19, evidenciando que el 13,1\% presentó una tasa de filtración glomerular menor de $60 \mathrm{ml} /$ $\mathrm{min} / \mathrm{m} 2$ al ingreso; además, mostró que los marcadores de daño renal como proteinuria, hematuria y creatinina elevada, así como el desarrollo de la insuficiencia renal aguda (IRA) se asociaron a mayor mortalidad hospitalaria. ${ }^{8}$ $\mathrm{Y}$, dos metaanálisis hicieron referencia que la enfermedad renal crónica (ERC) podría estar asociada con mayor riesgo de presentar infección severa. ${ }^{9,10}$ Otro autor reportó que los pacientes que requirieron ingreso a Unidad de Cuidados Intensivos (UCI) tuvieron mayor edad y mayores comorbilidades $(72,2 \%$ vs $37,3 \%, p<0,001)$, aunque al comparar la ERC entre los pacientes que fueron a UCI y aquellos que no requirieron tratamiento intensivo no se encontró diferencia significativa $(5,6 \%$ vs $2,0 \%, p=0,28)$ entre ambos grupos. ${ }^{11}$

Respecto a los pacientes en terapia sustitutiva renal, la Sociedad Española de Nefrología realizó el Registro COVID-19 encontrando que la gran mayoría fueron varones, con una edad media de $67 \pm 15$ años y además, mayor frecuencia en pacientes que recibían hemodiálisis en centro (63\%), seguida de los pacientes trasplantados (33\%) y en diálisis peritoneal (4\%). ${ }^{12}$ Este estudio también encontró una alta incidencia de ingreso hospitalario, con una mortalidad del $23 \%$, resaltando que del grupo de fallecidos, el $67,7 \%$ fue de los varones, el $69,5 \%$ se encontraban en hemodiálisis y el 90,1\% desarrolló neumonía y, a su vez, tuvieron mayor edad, más casos en hemodiálisis y mayor incidencia de neumonía que aquellos que sobrevivieron.

\section{RELACIÓN ECA/ECA-2: PAPEL EN LA ENFERMEDAD RENAL CRÓNICA Y COVID-19}

El sistema renina-angiotensina-aldosterona (RAS) juega un papel primordial en las enfermedades cardiovasculares y principalmente en la progresión de la ERC. La ECA2, una enzima expresada en el túbulo proximal, y con menor frecuencia en los glomérulos, permite disminuir la vasoconstricción, la retención de agua, la proliferación celular, el estrés oxidativo y tiene un efecto nefroprotector mediante la producción de angiotensina 1-7.

Se ha propuesto que, en condiciones normales, existe un equilibrio entre ECA y ECA-2, encontrándose que la relación ECA/ECA-2 se encuentra alterada en varias enfermedades y se ha postulado como un regulador del RAS renal.

En las patologías cardiovasculares hay un incremento de la relación ECA/ECA-2 que conduce a disfunción renal y estrés oxidativo y se ha correlacionado con elevación de presión arterial sistólica, glucosa y proteinuria en humanos. ${ }^{13}$

En pacientes con diabetes mellitus y nefropatía diabética se ha identificado niveles elevados de la relación ECA/ ECA-2 que podrían desempeñar un papel importante en el daño renal. ${ }^{4}$ De igual forma, en pacientes hipertensos con nefroangioesclerosis se evidenció que los niveles de ECA y ECA-2 intrarrenales participan en la patogénesis y progresión de la hipertensión y que el aumento de los niveles de ECA/ECA-2 se asocia a mayor glomeruloesclerosis $\mathrm{y}$ menor tasa de filtración glomerular. ${ }^{15}$ Otro estudio demostró que los pacientes en hemodiálisis (HD), tuvieron niveles más elevados de ECA/ECA-2 que aquellos en hemodiálisis sin enfermedad cardiovascular (ECV) y que en los controles sanos, a su vez, los niveles de ECA/ECA-2 se incrementaron durante la evaluación post hemodiálisis respecto a los valores pre diálisis. ${ }^{16}$

Al parecer, este aumento en la relación ECA/ECA-2 también jugaría un rol en la infección por COVID-19 generando peores resultados y esto es deducible ya que las comorbilidades como hipertensión, cardiopatía isquémica, insuficiencia renal crónica, fibrilación auricular o insuficiencia cardíaca se caracterizan por una relación ECA/ECA-2 elevada, de igual forma con la obesidad que ha sido asociada a mortalidad. ${ }^{13}$

\section{ESPECTRO CLÍNICO DEL COVID-19 EN DIÁLISIS ¿ES DISTINTO A LA POBLACIÓN GENERAL?}

En la población general se han descrito una serie de síntomas asociadas a la infección por COVID-19, destacando fiebre, tos y fatiga como los síntomas más frecuentes, aunque se han visto en pequeños porcentajes congestión nasal y conjuntival, mareo, dolor abdominal e incluso hemoptisis.3, 6, 17 Otros de los síntomas que resaltan dentro del espectro clínico de la infección por SARS-CoV-2 son la anosmia y disgeusia, aunque también se han reportado erupción cutánea y manifestaciones neurológicas, como accidente cerebro vascular y convulsiones. . $^{78-20}$

En adultos mayores se reportó a la fiebre y la tos seca como los más frecuentes, aunque se presentaron otras manifestaciones como disnea $(29,1 \%)$, mialgia o artralgia $(26,6 \%)$, cefalea $(4,9 \%)$, diarrea $(4,9 \%)$, dolor toráxico $(2 \%)$ y náuseas $(1,5 \%)$; sin embargo, no se encontró diferencia significativa al compararlos entre menores y mayores de 65 años. ${ }^{21}$ Un estudio descriptivo en pacientes críticamente 


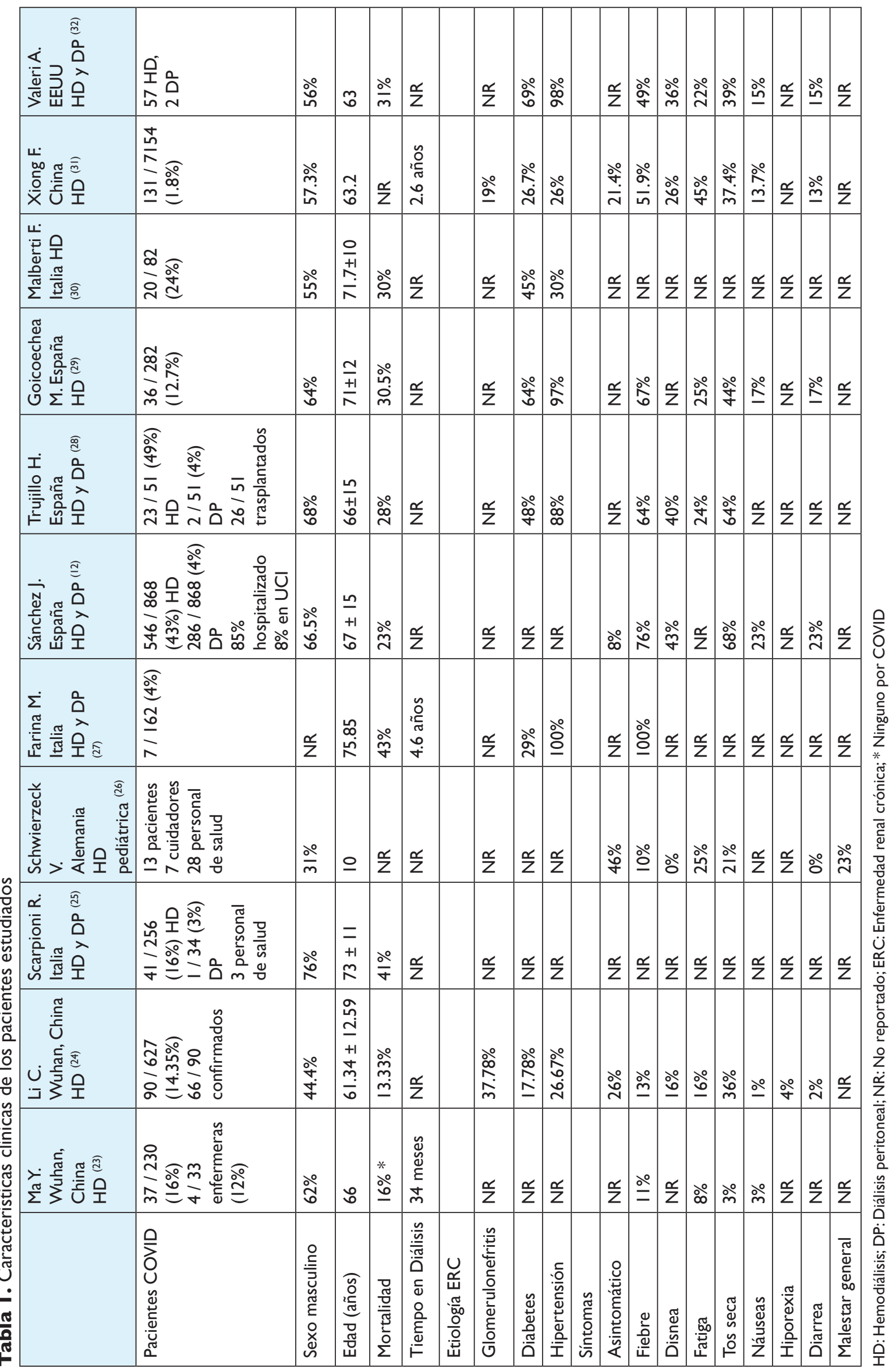


Tabla 2. Exámenes de los pacientes en diálisis con COVID

\begin{tabular}{|c|c|c|c|c|c|}
\hline & $\begin{array}{l}\text { Ma Y, et al. } \\
\text { Wuhan, China } \\
\text { Hemodiálisis }\end{array}$ & $\begin{array}{l}\text { Li C, et al. } \\
\text { Wuhan, China } \\
\text { Hemodiálisis }\end{array}$ & $\begin{array}{l}\text { Trujillo H, et al. } \\
\text { Madrid, España } \\
\text { Hemodiálisis y } \\
\text { Diálisis Peritoneal }\end{array}$ & $\begin{array}{l}\text { Goicoechea M. } \\
\text { España } \\
\text { Hemodiálisis }\end{array}$ & $\begin{array}{l}\text { Xiong F. } \\
\text { China } \\
\text { Hemodiálisis }\end{array}$ \\
\hline \multicolumn{6}{|l|}{ Laboratorio } \\
\hline Hemoglobina $(\mathrm{g} / \mathrm{dl})$ & 11.2 & 10.12 & $11.1 \pm 2$ & $10.6 \pm 1.4$ & 10.5 \\
\hline Creatinina $(\mathrm{mg} / \mathrm{dl})$ & NR & 7.85 & 5 & NR & NR \\
\hline Albúmina $(\mathrm{g} / \mathrm{dl})$ & 4.0 & 3.85 & $3.6 \pm 0.6$ & $3.7 \pm 0.4$ & 3.7 \\
\hline Leucocitos $\left(\times 10^{9} / \mathrm{L}\right)$ & 6.37 & $6.50 \pm 3.35$ & NR & NR & 5.0 \\
\hline Disminuido & $2(11 \%)$ & NR & NR & NR & NR \\
\hline Neutrófilos $\left(\times 10^{3} / \mathrm{L}\right)$ & 4.21 & $4.82 \pm 3.11$ & NR & NR & 3.9 \\
\hline Aumentado & $2(11 \%)$ & $\mathrm{NR}$ & NR & $\mathrm{NR}$ & NR \\
\hline Disminuido & $2(11 \%)$ & $\mathrm{NR}$ & NR & $\mathrm{NR}$ & NR \\
\hline Linfocitos $\left(\times 10^{9} / \mathrm{L}\right)$ & 0.85 & $0.95 \pm 0.41$ & NR & $0.79 \pm 0.47$ & 0.7 \\
\hline Aumentado & I (5\%) & NR & NR & NR & NR \\
\hline Disminuido & $13(68 \%)$ & NR & NR & NR & NR \\
\hline Plaquetas $\left(\times 10^{9} / \mathrm{L}\right)$ & 143 & $160.43 \pm 79.02$ & NR & $164 \pm 66$ & 144.2 \\
\hline Disminuido & $6(32 \%)$ & NR & NR & NR & NR \\
\hline PCR & NR & 6.98 & 8 & $9.6 \pm 7.5$ & NR \\
\hline Aumentada & $6(32 \%)$ & NR & NR & NR & NR \\
\hline $\mathrm{DHL}(\mathrm{U} / \mathrm{L})$ & NR & $184.60 \pm 51.96$ & $310 \pm 101$ & $235 \pm 58$ & NR \\
\hline Dimero D (ng/ml) & NR & NR & 1106 & 375 & NR \\
\hline \multicolumn{6}{|l|}{ Radiología (TAC) } \\
\hline Neumonía unilateral & $15(41 \%)$ & $12(18.46 \%)$ & NR & $7(19 \%)$ & NR \\
\hline Neumonía derecha & $12(57 \%)$ & NR & NR & NR & $7.1 \%$ \\
\hline Neumonía izquierda & $3(8 \%)$ & NR & NR & NR & $6.2 \%$ \\
\hline Neumonía bilateral & 22 (59\%) & $53(81.54 \%)$ & $16(64 \%)$ & NR & $86.7 \%$ \\
\hline Vidrio esmerilado & $23(62 \%)$ & $23(35.38 \%)$ & $15(60 \%)$ & $22(61 \%)$ & $82.1 \%$ \\
\hline
\end{tabular}

NR: no reportado; PCR: Proteína C reactiva; DHL: lactato deshidrogenasa;TAC:Tomografía axial computarizada

enfermos, con una media de edad de $64 \pm 18$ años, mostró a la tos y disnea como los síntomas más prevalentes, pero reportó una mortalidad elevada del $50 \%{ }^{(22)}$.

Se realizó una revisión de las manifestaciones clínicas encontradas en la población en diálisis, las cuales se resumen en la Tabla 1. Cabe resaltar, que la mayoría de estudios se realizaron en pacientes en hemodiálisis, encontrándose que el mayor porcentaje fueron del sexo masculino, requirieron menores cuidados en UCI y tuvieron una mortalidad con cifras que oscilaron entre $13,33 \%$ y $43,0 \% .^{23-32}$, aunque un estudio menciona que no hubo pacientes fallecidos a causa del COVID-19. Además, se encontró casos positivos en el personal de salud e incluso en los cuidadores de los pacientes, probablemente relacionado al hacinamiento propio de las unidades de hemodiálisis. Como era de esperar, la gran mayoría de pacientes fueron diabéticos (entre $17,78 \%$ y $64,0 \%$ ) e hipertensos (26,67\% a $100 \%)$. Dentro de los síntomas, es importante remarcar que entre el $8 \%$ y $46 \%$ de los evaluados fueron asintomáticos y, a diferencia de lo que se reporta en la población sin diálisis, otros autores encontraron una prevalencia del $40,5 \% .{ }^{33}$
Asimismo, hubo menor incidencia de fiebre, tos y fatiga34 aunque si resaltan los síntomas gastrointestinales. ${ }^{12,29}$ Algunos reportes de casos han mostrado que gran parte de los pacientes con enfermedad renal crónica en diálisis presentan neumonía, aunque no es severa. ${ }^{27,35,36}$

Los pacientes con COVID-19 suelen cursar con leucopenia $(9,1-33,7 \%)$, linfopenia $(35,3-82,1 \%)$, trombocitopenia (5\%-36,2\%), proteína $\mathrm{C}$ reactiva elevada $(60,7-86,3 \%)$, dímero D elevado (36,4\%-46,4\%), deshidrogenasa elevada $(27,4 \%-75,8 \%)$, creatina kinasa elevada (8\%$32,5 \%$ ), tiempo de protrombina prolongado (58\%), alanina aminotransferasa elevada (16,1\%-28,3\%), aspartato aminotransferasa incrementada (22,2\%-36,7\%), interleukina-6 elevada $(51,5 \%)$, ferritina sérica elevada $(62,6 \%)$, procalcitonina elevada $(5,5 \%-11,3 \%)$ y creatinina elevada $(1,9 \%-9,8 \%)$, aunque también se ha reportado anemia (51\%), leucocitosis (24\%), neutrofilia (38\%), hipoalbuminemia (98\%), hiperglicemia (52\%) y $\mathrm{PaFiO} 2$ disminuida. ${ }^{11,37,38}$

En los pacientes que requirieron UCI se encontró mayor neutrofilia, mayor linfopenia, mayor plaquetopenia, mayor 
nivel de dímero-D, mayor hipoalbuminemia y mayor DHL que aquellos que no ingresaron a UCI y, a su vez, tuvieron mayor síndrome de distrés respiratorio agudo, mayor falla cardíaca aguda, mayor injuria renal aguda, más choque e infección secundaria. ${ }^{39}$ En cuanto a los estudios radiológicos, la tomografía es el examen de elección, sobre todo en pacientes asintomáticos, encontrándose un patrón bilateral $(79 \%)$, periférico $(54 \%)$, mal definido $(81 \%)$ y opacificación en vidrio esmerilado $(65 \%)$, principalmente en el lóbulo inferior derecho, aunque también se ha reportado derrame pleural hasta en $8 \%$ de los casos. ${ }^{40,41} \mathrm{Se}$ ha propuesto el uso de la ecografía toráxica donde logra verse la presencia de líneas B difusas y consolidaciones subpleurales. ${ }^{42,43}$

En la tabla 2 se resumen los exámenes y patrones radiológicos encontrados en los pacientes en diálisis. No se encuentra mayor diferencia que lo hallado en pacientes que no reciben diálisis. Sin embargo, se debe resaltar un interesante hallazgo que muestra que la ferritina se elevaría hasta en un $275 \%$ en los pacientes que dieron positivo a COVID-19 durante la infección respecto a sus valores previos a la infección, e incluso se incrementaron al momento del diagnóstico en cinco pacientes asintomáticos, así como en los pacientes con síntomas. ${ }^{44}$ También, se ha visto que los pacientes en hemodiálisis con COVID-19 tienen menor recuento de células $\mathrm{T}$, Th, killers $\mathrm{T}, \mathrm{NK}$ y linfocitos B, así como IL-4, IL-6 y TNF $\alpha$ que el resto de infectados sin ERC, lo que contribuiría a que no se desarrolle tormenta de citoquinas. ${ }^{23,45}$

\section{CONCLUSIONES}

Dentro del grupo de riesgo de contraer COVID-19 se encuentran los pacientes con enfermedad renal crónica, principalmente en hemodiálisis, en quienes se ha encontrado una alteración de la relación ECA/ECA-2. Llama la atención la presencia de síntomas similares a la población en general, aunque con mayor presentación de síntomas gastrointestinales. Se debe realizar más estudios en pacientes en diálisis para definir el comportamiento de la enfermedad en esta población susceptible.

\section{REFERENCIAS BIBLIOGRÁFICAS}

1. Organización Mundial de la Salud. Coronavirus disease 2019(COVID-19). Situation Report - 209. Disponible en: https:// www.who.int/docs/default-source/coronaviruse/situation-reports/ 20200816-covid-19-sitrep-209.pdf?sfvrsn=5dde1 ca2_2

2. De la Serna F. Novedades en el sistema renina-angiotensina. Insuf Card. 2014;9(1):16-24

3. Guan W, Ni Z, Hu Yu, Liang W, Ou C, He J, et al. Clinical characteristics of coronavirus disease 2019 in China. $N$ Engl J Med. 2020;382(18):1708-1720. doi: 10.1056/NEJMoa2002032

4. Richardson S, Hirsch J, Narasimhan M, Crawford J, McGinn $\mathrm{T}$, Davidson K, et al. Presenting characteristics, comorbidities, and outcomes among 5700 patients hospitalized with COVID-19 in the New York City Area. JAMA. 2020:E1-E8. doi:10.1001/ jama.2020.6775

5. Chen T, Wu D, Chen H, Yan W, Yang D, Chen G, et al. Clinical characteristics of 113 deceased patients with coronavirus disease 2019: retrospective study. BMJ. 2020;368:m1091. doi: http://dx.doi. org/10.1136/bmj.m1091

6. Zhao XY, Xu XX, Yin HS, Hu QM, Xiong T, Tang YY, et al. Clinical characteristics of patients with 2019 coronavirus disease in a nonWuhan area of Hubei Province, China: a retrospective study. BMC Infectious Diseases. (2020) 20:311. doi: https://doi.org/10.1186/ s12879-020-05010-w

7. Aggarwal S, Garcia-Telles N, Aggarwal G, Lavie C, Lippi G, Henry B. Clinical features, laboratory characteristics, and outcomes of patients hospitalized with coronavirus disease 2019 (COVID-19): Early report from the United States. Diagnosis. 2020; 7(2): 91-96. doi: https://doi.org/10.1515/dx-2020-0046

8. Cheng Y, Luo R, Wang K, Zhang M, Wang Z, Dong L, et al. Kidney disease is associated with in-hospital death of patients with COVID-19. Kidney Int. 2020;97(5):829-838. doi:10.1016/j. kint.2020.03.005

9. Henry BM, Lippi G. Chronic kidney disease is associated with severe coronavirus disease 2019 (COVID-19) infection. Int Urol Nephrol 2020; 1-2. doi: 10.1007/s11255-020-02451-9

10. Wang X, Fang X, Cai Z, Wu X, Gao X, Min J, et al. Comorbid chronic diseases and acute organ injuries are strongly correlated with disease severity and mortality among COVID-19 Patients: A Systemic Review and Meta-Analysis. Research. 2020:2402961. doi: $10.34133 / 2020 / 2402961$

11. Wang $\mathrm{D}, \mathrm{Hu} \mathrm{B}, \mathrm{Hu} \mathrm{C}, \mathrm{Zhu} \mathrm{F}$, Liu X, Zhang J, et al. Clinical characteristics of 138 hospitalized patients with 2019 novel coronavirus-infected pneumonia in Wuhan, China. JAMA. 2020;323(11): 1061-1069. doi:10.1001/jama.2020.1585

12. Sánchez JE, Pérez M, Jiménez C, Blasco M, Cabezas CJ, Sevillano A, et al. Situación de la infección por SARS-CoV-2 en pacientes en tratamiento renal sustitutivo Informe del Registro COVID-19 de la Sociedad Española de Nefrología (S.E.N.). Nefrología. 2020;40(3):272-278. doi: https://doi.org/10.1016/j. nefro.2020.04.002

13. Pagliaro P, Penna C. ACE/ACE2 Ratio: A Key Also in 2019 Coronavirus Disease (Covid-19)? Front. Med. 2020; 7:335.doi: 10.3389/fmed.2020.00335

14. Mizuiri S, Hemmi H, Arita M, Ohashi Y, Tanaka Y, Miyagi M, et al. Expression of ACE and ACE2 in individuals with diabetic kidney disease and healthy controls. Am J Kidney Dis. 2008; 51: 613-623. doi: 10.1053/j.ajkd.2007.11.022

15. Wang G, Lai FM, Kwan BC, Lai KB, Chow KM, Li PK, et al. Expression of ACE and ACE2 in patients with hypertensive nephrosclerosis. Kidney Blood Press Res. 2011; 34: 141-149. doi: 10.1159/000324521

16. Chung-Wei Yang, Li-Che Lu, Chia-Chu Chang, Ching-Chang Cho, Wen-Yeh Hsieh, Chin-Hung Tsai, et al. Imbalanced plasma ACE and ACE2 level in the uremic patients with cardiovascular diseases and its change during a single hemodialysis session. Renal Failure. 2017; 39:1, 719-728. doi: 10.1080/0886022X.2017.1398665

17. Zhang JJ, Dong X, Cao XY, Yuan YD, Yang YB, Yan YQ. Clinical characteristics of 140 patients infected with SARSCoV-2 in Wuhan, China. Allergy. 2020;00:1-12. Doi: https://doi.org/10.1111/all.14238

18. Guan WJ, Liang WJ, Zhao Y, Liang HR, Chen ZS, Li YM, et al. Comorbidity and its impact on 1590 patients with Covid-19 in China: A Nationwide Analysis. Eur Respir J. 2020; in press (https:// doi.org/10.1183/13993003.00547-2020).

19. Mao L, Jin H, Wang M, Hu Y, Chen S, He Q, et al. Neurologic manifestations of hospitalized patients with coronavirus disease 2019 in Wuhan, China. JAMA Neurol. 2020: E1 - E8. doi:10.1001/ jamaneurol.2020.1127.

20. Li Z, Liu T, Yang N, Han D, Mi X, Li Y. Neurological manifestations of patients with COVID-19: potential routes of SARS-CoV-2 neuroinvasion from the periphery to the brain. Front Med. 2020:1-9. doi:https://doi.org/10.1007/s11684-020-0786-5.

21. Chen TL, Dai Z, Mo P, Li X, Ma Z, Song S, et al. Clinical characteristics and outcomes of older patients with coronavirus disease 2019 (COVID-19) in Wuhan, China (2019): a singlecentered, retrospective study. The Journals of Gerontology: 2020:131. Doi: https://doi.org/10.1093/gerona/glaa089

22. Bhatraju PK, Ghassemieh BJ, Nichols M, Kim R, Jerome KR, Nalla AK, et al. COVID-19 in critically ill patients in the Seattle region Case Series. NEJM. 2020: 1-11. Doi: 10.1056/NEJMoa2004500 
23. Ma Y, Diao B, Lv X, Zhu J, Liang W, Liu L, et al. 2019 novel coronavirus disease in hemodialysis (HD) patients: Report from one HD center in Wuhan, China. MedRxiv. 2020.02.24.20027201. doi: https://doi.org/10.1101/2020.02.24.20027201

24. Li C, Yonglong M, Can T, Dongdong M, Sheng W, Haifeng L, et al. An Analysis on the Clinical Features of MHD Patients with Coronavirus Disease 2019: A Single Center Study. Research Square. 2020. Doi: 0.21203/rs.3.rs-18043/v1

25. Scarpioni R, Manini A, Valsania T, De Amicis S, Albertazzi V, Melfa L, et al. Covid-19 and its impact on nephropathic patients: the experience at Ospedale "Guglielmo da Saliceto" in Piacenza. G Ital Nefrol. 2020; 2(4):1-5.

26. Schwierzeck V, König JC, Kühn J, Mellmann A, Correa-Martínez CL, Omran H, et al. First reported nosocomial outbreak of severe acute respiratory syndrome coronavirus 2 (SARS-CoV-2) in a pediatric dialysis unit. Clin Infect Dis. 2020; ciaa491. doi:10.1093/ $\mathrm{cid} / \mathrm{ciaa} 491$

27. Farina M, Barbisoni F, Bertacchini S, Borettaz I, Bucci R, Maggio M, et al. An account of the first hours of the Covid-19 epidemic at the Nephrology Unit in Lodi (Lombardy). G Ital Nefrol. 2020; 2(3): $1-5$.

28. Trujillo H, Caravaca-Fontán F, Sevillano Á, Gutiérrez E, Caro J, Gutiérrez E, et al. SARS-CoV-2 Infection in Hospitalized Patients with Kidney Disease. Kidney International Reports. (2020). doi: https://doi.org/10.1016/j.ekir.2020.04.024.

29. Goicoechea M, Sánchez-Cámara LA, Macías N, Muñoz de Morales A, González Rojas Á, Bascuñana A, et al. COVID-19: Clinical course and outcomes of 36 maintenance hemodialysis patients from a single center in Spain. Kidney International. (2020). doi: https:// doi.org/10.1016/j.kint.2020.04.031

30. Malberti F, Pecchini P, Marchi G, Foramitti M. When a nephrology ward becomes a COVID-19 ward: the Cremona experience. Journal of Nephrology. 2020; 1-4. Doi: fghttps://doi.org/10.1007/s40620020-00743-y.

31. Xiong F, Tang H, Liu L, Tu C, Tian JB, Lei CT, et al. Clinical Characteristics of and Medical Interventions for COVID-19 in Hemodialysis Patients in Wuhan, China. JASN. 2020;31(7)13871397. DOI: https://doi.org/10.1681/ASN.2020030354

32. Valeri A, Robbins-Juarez S, Stevens J, Ahn W, Rao M, Radhakrishnan J, et al. Presentation and Outcomes of Patients with ESKD and COVID-19. JASN. 2020, 31 (7) 1409-1415; DOI: https:// doi.org/10.1681/ASN.2020040470

33. Albalate M, Arribas P, Torres E, Cintra M, Alcázar R, Puerta M, et al. Alta prevalencia de COVID-19 asintomático en hemodiálisis. Aprendiendo día a día el primer mes de pandemia de COVID-19. Nefrologia. 2020;40(3):279-286. https://doi.org/10.1016/j. nefro.2020.04.005

34. Wu J, Li J, Zhu G, Zhang Y, Bi Z, Yu Y, et al. Clinical Features of Maintenance Hemodialysis Patients with 2019 Novel Coronavirus-Infected Pneumonia in Wuhan, China. CJASN. 2020;15(1) CJN.04160320; DOI: 10.2215/CJN.04160320
35. Ke C, Wang Y, Zeng X, Yang C, Hu C. 2019 novel coronavirus disease (COVID-19) in hemodialysis patients: a report of two cases. Clinical Biochemistr. (2020), doi: https://doi.org/10.1016/j. clinbiochem.2020.04.008

36. Wang R, Liao C, He H, Hu C, Wei Z, Hong Z, et al. COVID-19 in Hemodialysis Patients: A Report of 5 Cases. Am J Kidney Dis. XX(XX):1-3. doi: https://doi.org/10.1053/j.ajkd.2020.03.009.

37. Ge H, Wang X, Yuan X, Xiao G, Wang C, Deng T, et al. The epidemiology and clinical information about COVID-19. Eur J Clin Microbiol Infect Dis. 2020. Doi: https://doi.org/10.1007/s10096020-03874-Z

38. Chen N, Zhou M, Dong X, Qu J, Gong F, Han Y, et al Epidemiological and clinical characteristics of 99 cases of 2019 novel coronavirus pneumonia in Wuhan, China: a descriptive study. Lancet. 2020;395(10223):507-513. Doi: https://doi.org/10.1016/ S0140-6736(20)30211-7

39. Huang C, Wang Y, Li X, Ren L, Zhao J, Hu Y, et al. Clinical features of patients infected with 2019 novel coronavirus in Wuhan, China. Lancet. 2020;395(10223):497-50. Doi: https://doi.org/10.1016/ S0140-6736(20)30183-5

40. Shi H, Han X, Jiang N, Cao Y, Alwalid O, Gu J, et al. Radiological findings from 81 patients with COVID-19 pneumonia in Wuhan, China: a descriptive study. Lancet Infect Dis. 2020 04; 20(4):425434. Doi: https://doi.org/10.1016/S1473-3099(20)30086-4

41. Ye Z, Zhang Y, Wang Y, Huang Z, Song B. Chest CT manifestations of new coronavirus disease 2019 (COVID-19): a pictorial review. Eur Radiol. (2020). https://doi.org/10.1007/s00330-020-06801-0

42. Lomoro P, Verde F, Zerboni F, Igino S, Claudia S, Camilla B, et al. COVID-19 pneumonia manifestations at the admission on chest ultrasound, radiographs, and CT: single-center study and comprehensive radiologic literature review. Eur J Radiol Open. 2020; 7: 100231. doi: 10.1016/j.ejro.2020.100231

43. Peng QY, Wang XT, Zhang LN. Findings of lung ultrasonography of novel corona virus pneumonia during the 2019-2020 epidemic. Intensive Care Med. 2020 Mar 12. Doi: 10.1007/s00134-020-05996-

44. Bataille S, Pedinielli N, Bergougnioux JP. Could ferritin help the screening for COVID-19 in hemodialysis patients? Kidney Int. 2020 Apr 22. doi: 10.1016/j.kint.2020.04.017

45. Florit EA, Diekmann F. ¿Es la hiporrespuesta inmune de la uremia un factor de protección contra la neumonía grave por COVID-19? Nefrología. 2020. Doi: https://doi.org/10.1016/j.nefro.2020.05.002

Conflicto de interés: Ninguno, según el autor.

Financiamiento: Por el autor.

\section{CORRESPONDENCIA}

Cristhian Adolfo Vizcarra Vizcarra

cristhianvizcarra12@hotmail.com

ORCID: https://orcid.org/0000-0003-1068-7793

Fecha de recepción: 16-09-2020.

Fecha de aceptación: 16-1|-2020. 